EPJ Web of Conferences 70, 00014 (2014)

DOI: 10.1051/epjconf/20147000014

(C) Owned by the authors, published by EDP Sciences, 2014

\title{
Fermiophobic Higgs boson and supersymmetry
}

\author{
Antonio Racioppi ${ }^{\mathrm{a}}$ \\ ${ }^{1}$ National Institute of Chemical Physics and Biophysics, Ravala 10, Tallinn 10143, Estonia
}

\begin{abstract}
If a light Higgs boson with mass $125 \mathrm{GeV}$ is fermiophobic, or partially fermiophobic, then the MSSM is excluded. The minimal supersymmetric fermiophobic Higgs scenario can naturally be formulated in the context of the NMSSM that admits $Z_{3}$ discrete symmetries. We show that in the fermiophobic NMSSM the radiative Higgs boson branchings to $\gamma \gamma, \gamma Z$ can be modified compared to the fermiophobic and ordinary standard model predictions.
\end{abstract}

\section{Introduction}

The TeVatron [1] and LHC experiments presented their temporary results [2,3] on searches for the Higgs boson at the Moriond 2012 conference [4]. While on average the data was consistent with the standard model (SM) Higgs boson with mass $125 \mathrm{GeV}$, interesting anomalies started to emerge that may signal unexpected new physics in the Higgs sector. The most interesting of them was a local $3 \sigma$ level excess in searches for the fermiophobic (FP) Higgs boson [5] in $\gamma \gamma$ final states both in the ATLAS and CMS experiments [6]. This signaled that there could be an anomalously large contribution in the observed $\gamma \gamma$ excess coming from the vector-boson fusion (VFB) Higgs production mechanism. Indeed, the relative weight of the latter and the associate production with $W, Z(\mathrm{VH})$ was enhanced with respect to the SM dominant gluon-gluon fusion channel $(\mathrm{ggF})$ in the FP high- $p_{T}$ selections applied by the CMS and ATLAS. This anomaly was accompanied by a deficit of $W W^{*}$ compared with the SM in all experiments.

The Higgs boson mass $M_{h} \approx 125 \mathrm{GeV}$ is peculiar in several ways. In the context of FP Higgs boson, there is an accident that at the 7-8 TeV LHC the Higgs boson signal rate in the $\gamma \gamma$ channel, $\sigma \times B R$, happens to be equal to the SM one in the vicinity of this Higgs mass value [7]. Therefore a global fit to all available collider data was performed in order to determine which Higgs boson scenario is currently favored [8], improving and extending similar pre-Moriond fits [9]. A purely FP Higgs boson gave a fit to data almost as good as the SM one, but with very different predictions for the signal rates at the LHC. A partially FP Higgs boson, however, gave a significantly better fit to data than the SM [8] and partial fermiophobia is exactly what is expected to happen when considering the FP Higgs boson scenario as an effective low energy theory in the context of quantum field theory [1].

The $125 \mathrm{GeV}$ Higgs boson is also unsatisfactory, because this mass is below the SM vacuum stability bound in case new physics appears only above the scale of gauge coupling unification. The vacuum stability can be made consistent with the $125 \mathrm{GeV}$ Higgs mass by extending the scalar sector

\footnotetext{
ae-mail: antonio.racioppi@kbfi.ee
} 
with dark matter candidates [1]. But the simplest solution is provided by (partial) fermiophobia. Because the vacuum instability is caused by the large top Yukawa coupling in the SM, reducing its value makes the hinted Higgs mass compatible with the GUT scale.

It is well known that the existence of the Higgs boson rises a question of why the electroweak scale is so much smaller than the Planck scale. The most elegant solution to that problem is given by supersymmetry (SUSY). However, direct and indirect collider bounds, cosmological dark matter abundance and constraints from dark matter direct detection experiments together with a Higgs boson mass of $125 \mathrm{GeV}$ impose stringent constraints on SUSY scale in most popular SUSY models - the MSSM $[1,1]$ and NMSSM $[1,1]$. The constraint $M_{\text {SUSY }}>1 \mathrm{TeV} \gg \mathrm{M}_{\mathrm{Z}}$ reintroduces severe fine tuning to theory, known as the little hierarchy problem [1], that makes SUSY as a solution to the hierarchy problem unnatural.

If the Higgs boson turns out to be fermiophobic, some SUSY models are in even more serious trouble. We show that fermiophobia and a Higgs boson mass at $125 \mathrm{GeV}$ together exclude all versions of the MSSM independently of any model detail. This is because in the MSSM the upper bound on the tree level Higgs boson mass is $M_{Z}$, and large radiative corrections, dominated by stop contributions, are needed to reach $125 \mathrm{GeV}$. Fermiophobia removes the dominant stop loops as they are induced by Yukawa couplings. There might be large trilinear scalar couplings, the $A$-terms, which may trigger electroweak symmetry breaking [1], but cannot increase the Higgs boson mass.

Nevertheless, SUSY models with additional tree level contributions to the Higgs boson mass, such as the NMSSM, are viable fermiophobic SUSY theory candidates. In fact fermiophobia can cure some SUSY problems and make it more compatible with experimental data. Firstly, the fine tuning problem of SUSY, also coming from loop contributions to the Higgs boson mass squared, is now induced by gauge couplings, improving the fine tuning by a factor of $N_{c} y_{t}^{4} / g^{4} \sim 25$. This improvement completely removes the little hierarchy problem. SUSY masses of order 2-3 TeV become completely natural. Even the split SUSY [1] would become viable. Secondly, SUSY flavor and CP problems are improved by removing (or decreasing) the Yukawa couplings and by allowing also squark and slepton masses to be at a few-TeV scale. Thirdly, the absence of constraints from $b \rightarrow s \gamma$ and $B_{s} \rightarrow \mu \mu$ [1] allows the charged Higgs boson to be light, opening again the possibility for its discovery at the LHC. Finally, the constraints on dark matter are relaxed. Because neutralino elastic scattering off nuclei is dominated by tree level Higgs boson exchange, this process would be suppressed and the prospects for dark matter discovery at the XENON100 are decreased in this scenario [2]. As the SUSY scale could now be large, higgsino or wino relic abundance would become a natural explanation to the dark matter of the Universe.

The obvious question in any FP Higgs boson scenario is what is the alternative mechanism for generating the observed fermion masses. Because the top quark mass is so large, it cannot be generated radiatively. The most plausible scenario for generating such large fermion masses is strong dynamics above the electroweak scale [2]. In such a scenario both the composite Higgs boson fermiophobia and fermion masses might originate from the same new physics. A generic prediction of strong electroweak symmetry breaking scenarios, including composite Higgs models, is the appearance of new resonances at $2-3 \mathrm{TeV}$. In the following, we assume that such or any other new physics scenario above the electroweak scale generates the top quark mass.

\section{Fermiophobic supersymmetry}

We formulate the first attempt of a supersymmetric FP Higgs boson theory in the context of the MSSM. However, we are going to show that the Higgs boson mass $M_{h} \approx 125 \mathrm{GeV}$ is by far too large to be generated in the FP MSSM since loop corrections from the top Yukawa coupling are absent. 
The dominant SUSY loop contribution to the Higgs mass in the FP MSSM comes from the large trilinear $A$-term, but this contribution is always negative. Thus the FP MSSM is definitely excluded on phenomenological grounds. In order to rescue supersymmetry, we show that the NMSSM offers a natural framework to formulate a supersymmetric FP Higgs scenario consistent with experimental results.

\subsection{Fermiophobic MSSM}

The well known MSSM superpotential is

$$
\mathcal{W}=y_{u} Q H_{u} u^{c}+y_{d} H_{d} Q d^{c}+y_{e} H_{d} L e^{c}+\mu H_{u} H_{d},
$$

where $y_{u}, y_{d}$ and $y_{e}$ are the up quark, down quark and charged lepton Yukawa couplings. Following the LHC hints for a FP Higgs, we study its implications without trying to explain the suppression of Yukawa couplings in the context of MSSM. Thus, for simplicity, we just take $y_{u}=y_{d}=y_{e}=0$.

In the MSSM the tree level Higgs boson mass has the well known upper bound $M_{h}^{\text {tree }}<M_{Z}$. This comes from the fact that in SUSY the Higgs quartic couplings are generated by gauge couplings via the $D$-terms. As the Higgs boson quartic coupling is the only free parameter in the SM Higgs sector, in the MSSM there is no freedom to tune the tree level Higgs boson mass. To be consistent with experimental data, in the MSSM very large positive loop corrections to $M_{h}^{2}$ must be generated. Those loop corrections are dominated by top squark contributions that are induced by the top Yukawa coupling $y_{t}[2]$,

$$
\Delta M_{h}^{2}=3 y_{t}^{4} \frac{v^{2} \sin ^{4} \beta}{8 \pi^{2}}\left[\log \frac{M_{S}^{2}}{m_{t}^{2}}+\frac{X_{t}^{2}}{M_{S}^{2}}\left(1-\frac{X_{t}^{2}}{12 M_{S}^{2}}\right)\right],
$$

where $M_{S}$ is the average stop mass, $\tan \beta=v_{u} / v_{d}, v^{2}=v_{u}^{2}+v_{d}^{2}$, and $X_{t}$ is the stop mass mixing parameter

$$
X_{t}=A_{t}-\mu \cot \beta=\frac{a_{t}}{y_{t}}-\mu \cot \beta,
$$

where $a_{t}$ is the trilinear coupling of the soft term $a_{t} \tilde{Q} H_{u} \tilde{u}^{c}$. In order to achieve the Higgs boson mass indicated by the LHC experiments, $M_{h} \approx 125 \mathrm{GeV}$, (2) implies that the stop masses must exceed TeV scale. In the FP MSSM the dominant stop contribution is absent since we take $y_{t} \rightarrow 0$. Therefore

$$
\Delta M_{h}^{2}=-\frac{3 v^{2} \sin ^{4} \beta}{8 \pi^{2}} \frac{a_{t}^{4}}{12 M_{S}^{4}},
$$

that is negative. As the chargino loop contributions to the Higgs boson mass are of order few $\mathrm{GeV}$, in the FP MSSM the Higgs boson mass $125 \mathrm{GeV}$ is not achievable. Independently of model details, the FP MSSM is excluded by the Higgs boson mass.

\subsection{Fermiophobic NMSSM}

NMSSM is the next to minimal supersymmetric standard model whose particle content is extended by a gauge singlet chiral superfield $S$ (for reviews and references therein see [1]). The original motivation for the NMSSM was to explain why the MSSM superpotential parameter $\mu H_{u} H_{d}$ is of the same order as the soft SUSY breaking parameters. In addition, in the NMSSM the Higgs bosons obtain tree level mass not determined by the $D$-terms, thus allowing larger Higgs masses than $M_{Z}$. To achieve those goals, usually the most general NMSSM is constrained by imposing an additional $Z_{3}$ symmetry in 
addition to the $R$-parity. Those properties make the NMSSM our prime candidate for the minimal FP SUSY model. The superpotential of the FP NMSSM that we would like to obtain is given by

$$
\mathcal{W}=\lambda S H_{u} H_{d}+\frac{k}{3} S^{3}
$$

together with the following soft SUSY breaking terms

$$
\mathcal{L}_{\text {soft }}=-\left(m_{h_{u}}^{2} h_{u}^{\dagger} h_{u}+m_{h_{d}}^{2} h_{d}^{\dagger} h_{d}+m_{s}^{2} s^{\dagger} s\right)-\left(a_{\lambda} s h_{u} h_{d}+\frac{1}{3} a_{k} s^{3}+\text { h.c. }\right),
$$

where $s$ stands for the scalar component of the singlet chiral superfield $S$.

Thus we have to forbid the $\mu H_{u} H_{d}, S, S^{2}, y_{u} Q H_{u} u^{c}, y_{d} H_{d} Q d^{c}, y_{e} H_{d} L e^{c}$ terms by imposing an additional $Z_{3}$ symmetry and appropriately choosing the corresponding charges. The easiest configuration is $X_{H_{u}}=X_{H_{d}}=X_{S}=1$ and $X_{\text {fermion }}=0$. For a more detailed discussion we remand to [2].

The Yukawa couplings for the first two generations could be radiatively generated but not for the top quark. Therefore we have to assume that the significant amount of third generation fermion masses should come from some additional mechanism. The prime candidate for such a mechanism is some strong dynamics above $2-3 \mathrm{TeV}$ scale.

\section{Model setup and mass eigenstates}

The FP NMSSM scalar potential, and corresponding mass matrices are the same as the usual NMSSM, so in the following we are going to give only the main details about mass eigenstates and eigenvalues. We remand to our paper [2] and to NMSSM reviews [1, 1] for a more detailed discussion.

We start considering the CP-even Higgs bosons sector. It can be shown [2] that the parameters choice

$$
\begin{aligned}
\tan \beta & =1, \\
k & =\lambda, \\
a_{\lambda} & =0,
\end{aligned}
$$

allows no mixing between $s$ and $h_{u, d}^{0}$. Notice that $\tan \beta=1$ is allowed in this model because no constraints occur from the scalar potential minimization nor from the Yukawa sector. Therefore this choice is the most natural one. Adopting, for simplicity, the choice in eqs. (7)-(9) and requiring, of course, $v_{u} \neq 0, v_{d} \neq 0, v_{S} \neq 0$, the CP-even Higgs bosons square mass matrix, in the basis $\left(h_{d R}^{0}, h_{u R}^{0}, s_{R}\right)$, is

$$
M_{S}^{2}=\left(\begin{array}{ccc}
\frac{1}{2}\left(M_{Z}^{2}+v_{S}^{2} \lambda^{2}\right) & \frac{1}{2}\left(\left(v-v_{S}\right)\left(v+v_{S}\right) \lambda^{2}-M_{Z}^{2}\right) & 0 \\
\frac{1}{2}\left(\left(v-v_{S}\right)\left(v+v_{S}\right) \lambda^{2}-M_{Z}^{2}\right) & \frac{1}{2}\left(M_{Z}^{2}+v_{S}^{2} \lambda^{2}\right) & 0 \\
0 & 0 & 2 v_{S}^{2} \lambda^{2}+\frac{a_{k} v_{S}}{\sqrt{2}}
\end{array}\right)
$$

where $M_{Z}^{2}=\frac{1}{4}\left(g_{1}^{2}+g_{2}^{2}\right) v^{2}$. The corresponding eigenvectors and eigenvalues are

$$
\begin{array}{rlrl}
h & =\frac{1}{\sqrt{2}}\left(h_{d R}^{0}+h_{u R}^{0}\right), & & M_{h}^{2}=\frac{\lambda^{2} v^{2}}{2} \simeq 125 \mathrm{GeV}, \\
H & =\frac{1}{\sqrt{2}}\left(h_{d R}^{0}-h_{u R}^{0}\right), & M_{H}^{2}=M_{Z}^{2}+\frac{1}{2} \lambda^{2}\left(2 v_{S}^{2}-v^{2}\right), \\
s_{R}=s_{R}, & M_{s_{R}}^{2}=\frac{a_{k} v_{S}}{\sqrt{2}}+2 \lambda^{2} v_{S}^{2},
\end{array}
$$


Since we would like to identify one of the $\mathrm{CP}$-even eigenstates with the $125 \mathrm{GeV}$ resonance hinted by the LHC, it has to be made of doublets. In the notation of the MSSM, the Higgs mixing angle is given by $\alpha=-\pi / 4=\beta-\pi / 2$. In that case $H$ does not have any direct tree level coupling to $W W$ and $Z Z$ that explains why the LHC does not see presently any other resonance, but the lightest one at $125 \mathrm{GeV}$.

We skip the CP-odd Higgs bosons because they are not relevant for our discussion, and we give the charged Higgs boson mass and eigenstate

$$
\begin{aligned}
M_{H^{ \pm}}^{2} & =M_{W}^{2}+\frac{1}{2} \lambda^{2}\left(2 v_{S}^{2}-v^{2}\right), \\
H^{+} & =\frac{1}{\sqrt{2}}\left(h_{u}^{+}+h_{d}^{-*}\right) .
\end{aligned}
$$

For what concerns neutralinos and charginos mass matrices, the analysis is the same as the NMSSM, up to the substitutions (7)-(9). Therefore we remand to our paper [2] and to NMSSM reviews $[1,1]$ for more details.

\section{Radiative Higgs boson decays}

The model we have chosen to work with leaves the FP Higgs boson decays to $W W^{*}$ and $Z Z^{*}$ final states at tree level unaffected compared to the FP SM predictions. Because fermiophobia by itself is able to explain the observed deficit in $W W^{*}$ channel [7], the choice of a SUSY model is motivated by a maximized $\gamma \gamma$ rate. Deviations in $\gamma \gamma$ rate may happen due to the presence of extra particles in the loop. Because in the FP Higgs scenario the flavor physics constraints on charged Higgs masses are largely removed and chargino could be light, those particles can be as light as their present lower bounds from LEP II.

The free parameters at the EW scale which are relevant for our analysis are the following: the gaugino masses $M_{1}$ and $M_{2}$, the $\mu$ term given by $\mu \equiv \lambda v_{S} / \sqrt{2}$, the $\operatorname{sign}\left(\mu M_{1}\right)$ and $\operatorname{sign}\left(\mu M_{2}\right)$. In the present model, the mass of the charged Higgs is fixed once the value of $\mu$ is given, see Eq.(14). Moreover, we have chosen the convention of keeping $M_{2}$ positive, and allowing $\operatorname{sign}(\mu)$ to vary. We have set $M_{h}=125 \mathrm{GeV}, M_{1}=100 \mathrm{GeV}$ and $\sqrt{s}=7 \mathrm{TeV}$.

Then, $|\mu|, \operatorname{sign}(\mu)$ and $M_{2}$ are free parameters. We chose to re-express $|\mu|$ and $M_{2}$ as functions of two physical mass parameters: the charged Higgs mass $\left(M_{H^{+}}\right)$and the lightest chargino mass $\left(M_{\chi_{L}^{+}}\right)$, as follows

$$
\begin{aligned}
|\mu| & =\frac{\sqrt{M_{h}^{2}+\left(M_{H^{+}}^{2}-M_{W}^{2}\right) \sin ^{2}(2 \beta)-M_{Z}^{2} \cos ^{2}(2 \beta)}}{\sqrt{2}} \\
M_{2} & =\frac{ \pm \sqrt{2} M_{\chi_{L}^{+}} \sqrt{4 M_{W}^{2}\left(\mu^{2}-M_{\chi_{L}^{+}}^{2}\right)+2\left(M_{\chi_{L}^{+}}^{2}-\mu^{2}\right)^{2}+M_{W}^{4}(1-\cos 4 \beta)}-2 \mu M_{W}^{2} \sin 2 \beta}{2\left(M_{\chi_{L}^{+}}^{2}-\mu^{2}\right)} .
\end{aligned}
$$

There are two different values of the gaugino mass $M_{2}$, corresponding to the same lightest chargino mass. For convention $M_{2}>0$ and, for each $\operatorname{sign}(\mu)$ and $|\mu|$ value, only one of the two solutions is allowed. Finally, we recall that the $\lambda$ parameter is determined by inverting (11).

The corresponding decay widths for the radiative induced decays $h \rightarrow \gamma \gamma$ and $h \rightarrow Z \gamma$ of the lightest CP even Higgs boson $h$, in the framework of pure FP NMSSM model, are reported in our paper [2]. 

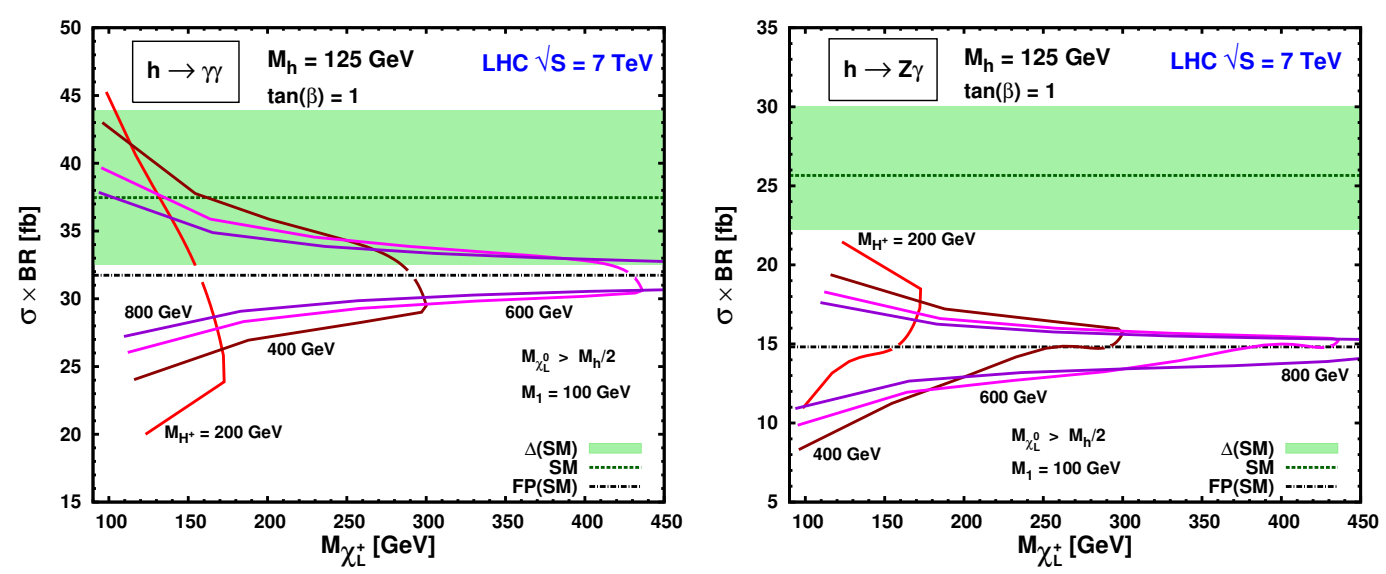

Figure 1. Radiatively induced signal rates of $125 \mathrm{GeV}$ FP NMSSM Higgs decays $h \rightarrow \gamma \gamma$ (left) and $h \rightarrow Z \gamma$ (right) at the $7 \mathrm{TeV}$ LHC as functions of the lightest chargino $\chi_{L}^{+}$mass $\left(M_{\chi_{L}^{+}}\right)$for several charged Higgs boson $H^{+}$ masses $\left(M_{H^{+}}\right)$as indicated in figures and for $\tan \beta=1$. The SM central value prediction (dashed line) together with $1 \sigma$ uncertainty band $\Delta(S M)$ and the FP SM Higgs prediction are also shown. The lines above (below) the FP SM line correspond to $\operatorname{sign}\left(M_{2} \mu\right)>0\left(\operatorname{sign}\left(M_{2} \mu\right)<0\right)$ for $h \rightarrow \gamma \gamma$ and to $\operatorname{sign}\left(M_{2} \mu\right)<0\left(\operatorname{sign}\left(M_{2} \mu\right)>0\right)$ for $h \rightarrow Z \gamma$.

In order to avoid a large tree-level Higgs decay into an invisible sector [2], that would destroy the potential enhancement of the Higgs decay into $\gamma \gamma$ [8], we will require that the mass of the lightest neutralino $\left(M_{\chi_{L}^{0}}\right)$, which is the lightest supersymmetric state in our scenario, is heavier than half of the Higgs mass. Then, due to $R$-parity, all other Higgs decays into two generic neutralino states $h \rightarrow \chi_{i}^{0} \chi_{j}^{0}$, including the case in which one or both are virtual states, will automatically vanish. In addition, we also require the lower bound on the chargino mass to be $90 \mathrm{GeV}$.

Taking into account the results obtained so far, we have computed the $h \rightarrow \gamma \gamma$ and $h \rightarrow Z \gamma$ signal rates for the FP Higgs boson in the FP NMSSM. We present our results in Fig. 1 where we plot the signal rates of those processes as functions of the lightest chargino mass $M_{\chi_{L}^{+}}$for different charged Higgs boson masses as indicated in the figure. The SM predictions together with their uncertainties and the FP SM predictions are also presented. The $1 \sigma$ (green) band corresponds to the theoretical uncertainty on the SM production cross section by gluon-gluon fusion. We have not included the uncertainty band on the SM FP line since the corresponding theoretical uncertainty due to the VBF cross section is quite small and can be neglected in this context.

From Fig. 1, we can see that for fixed chargino mass there are always two solutions for the oneloop SUSY amplitudes corresponding to $h \rightarrow \gamma \gamma$ and $h \rightarrow Z \gamma$ decays. This can be understood as follows. For values of $M_{\chi_{L}^{+}}$below the intersection point with the FP(SM) line, the double solution is mainly due to the sign of $\mu$ that controls the relative sign of the SUSY amplitude with respect to the SM one. The lines above (below) the FP SM line correspond to $\operatorname{sign}\left(M_{2} \mu\right)>0\left(\operatorname{sign}\left(M_{2} \mu\right)<0\right)$ for $h \rightarrow \gamma \gamma$ and to $\operatorname{sign}\left(M_{2} \mu\right)<0\left(\operatorname{sign}\left(M_{2} \mu\right)>0\right)$ for $h \rightarrow Z \gamma$.

However, for values of $M_{\chi_{L}^{+}}$above the intersection point, the sign of $\mu$ is fixed and the double solution corresponds to the fact that in the loop run two non-degenerates values of heavy chargino states at fixed $M_{\chi_{L}^{+}}$. The kink point corresponds to the case where the two solutions for the heavy chargino masses coincide. As we can see, there is a non-decoupling effect of the SUSY contribution 
to the loop $\gamma \gamma$ and $Z \gamma$ decay amplitudes in correspondence of the maximum value for the lightest chargino mass.

The absence of points in the half-plane above (below) the FP(SM) line for the curve corresponding to $M_{H^{+}}=200 \mathrm{GeV}$ in the case of $h \rightarrow \gamma \gamma(h \rightarrow Z \gamma)$, is due to the lightest neutralino mass constraint $M_{\chi_{L}^{0}}>M_{h} / 2$ and depends on our choice for $M_{1}=100 \mathrm{GeV}$.

As seen in the Fig. 1, the FP NMSSM signal rates can be both bigger or smaller than the FP SM predictions. For very light sparticles the total rate in $\gamma \gamma$ channel can even exceed the SM prediction. On the other hand, the after Moriond fits indicated that the LHC observed fewer $\gamma \gamma$ events than predicted by the pure FP SM [8]. That result could be easily explained in the FP NMSSM since also rate reductions of as much as $50 \%$ are possible for the chosen parameters.

\section{Conclusions}

If there is a signal of a fermiophobic, or partially fermiophobic, Higgs boson with mass $M_{h}=125$ $\mathrm{GeV}$, the fundamental idea of supersymmetry, as it is implemented in the MSSM, is in trouble and must be revised. In particular, we have shown that the MSSM with vanishing or strongly suppressed Yukawa couplings is ruled out, independently of the particular supersymmetry breaking mechanism. Indeed, due to the absence of Yukawa couplings the usual (large) logarithmic corrections to the Higgs mass, induced by the scalar particles running in the loops, are absent and the upper bound on the Higgs mass is very close the $M_{Z}$ mass.

In order to rescue supersymmetry, we show that a viable model beyond MSSM could be the NMSSM, where the absence of tree-level Yukawa couplings in the superpotential is guaranteed by the addition of a $Z_{3}$ discrete symmetry.

In this framework, we consider the particular NMSSM case in which the mixing of the singlet with doublet Higgs fields is absent in the CP-even sector and at tree level the lightest Higgs boson is exactly SM-like. We analyzed the predictions of this scenario for a $M_{h}=125 \mathrm{GeV}$ Higgs at the LHC. We show that the predictions for the one-loop Higgs boson branching fractions and production rates in $\gamma \gamma$ and $Z \gamma$ can be sizably modified with respect to the FP SM model, allowing a better fit to after Moriond collider data. However, the tree-level Higgs decay channels into $W W^{*}$ and $Z Z^{*}$ remain unaffected if the mixing between the singlet and doublet Higgs fields is absent. Relaxing this last condition, and so adding a new free parameter, the Higgs coupling to weak gauge boson $W W$ and $Z Z$ can be modified, and a suppression of the rates for $h \rightarrow W W^{*}$ and $h \rightarrow Z Z^{*}$ with respect to the pure FP model expectations can be achieved.

Finally, we would like to stress that the FP NMSSM offers a new arena for SUSY phenomenology at the LHC. In particular, most of the previous analysis on SUSY particle searches should be revised in the light of the fact that the large top-Yukawa coupling is absent or strongly suppressed.

\section{Acknowledgements}

The author acknowledges the ESF JD164 contract for financial support.

\section{References}

[1] The TEVNPH Working Group, f. t. CDF and D0 Collaborations, arXiv:1203.3774 [hep-ex]. CDF Collaboration, CDF Note 10806. D0 Collaboration, D0 Note 6303. Talk at the Moriond 2012 EW session. 
[2] ATLAS Collaboration, arXiv:1202.1414 [hep-ex], arXiv:1202.1415 [hep-ex], ATLAS-CONF2012-012, ATLAS-CONF-2012-014, ATLAS-CONF-2012-015, arXiv:1202.1408 [hep-ex].

[3] CMS Collaboration, arXiv:1202.1487 [hep-ex]. CMS-PAS-HIG-12-001. CMS-PAS-HIG-11025, 2011. arXiv:1202.1489 [hep-ex]. CMS-PAS-HIG-11-024, arXiv:1202.4083. CMS-PASHIG-12-007. CMS-PAS-HIG-11-031. arXiv:1202.1488 [hep-ex].

[4] D0 Collaboration talk, CDF Collaboration talk, and TeVatron combination talk at Moriond 2012. ATLAS Collaboration talk, CMS Collaboration talk at Moriond 2012. Searches for the BSM Higgs boson: TeVatron combination talk, ATLAS and CMS Collaboration talks at Moriond 2012. CMS-PAS-HIG-12-001. CMS-PAS-HIG-12-006. http://indico.in2p3.fr/ conferenceOtherViews.py?view $=$ standard\&confId=6001

[5] J. L. Basdevant, E. L. Berger, D. Dicus, C. Kao and S. Willenbrock, Phys. Lett. B 313, 402 (1993) [arXiv:hep-ph/9211225]. V. D. Barger, N. G. Deshpande, J. L. Hewett and T. G. Rizzo, [arXiv:hep-ph/9211244]. P. Bamert and Z. Kunszt, Phys. Lett. B 306, 335 (1993) [arXiv:hepph/9303239]. H. Pois, T. J. Weiler and T. C. Yuan, Phys. Rev. D 47, 3886 (1993) [arXiv:hepph/9303277]. A. Stange, W. J. Marciano and S. Willenbrock, Phys. Rev. D 49, 1354 (1994) [arXiv:hep-ph/9309294]. M. A. Diaz and T. J. Weiler, arXiv:hep-ph/9401259. A. G. Akeroyd, Phys. Lett. B 368, 89 (1996) [arXiv:hep-ph/9511347]. A. G. Akeroyd, Phys. Lett. B 368, 89 (1996) [hep-ph/9511347]. A. G. Akeroyd, J. Phys. G G 24, 1983 (1998) [hep-ph/9803324]. A. Barroso, L. Brucher and R. Santos, Phys. Rev. D 60 (1999) 035005 [hep-ph/9901293]. L. Brucher and R. Santos, Eur. Phys. J. C 12 (2000) 87 [hep-ph/9907434].

[6] CMS Collaboration, CMS-PAS-HIG-12-002. CMS Collaboration, CMS-PAS-HIG-12-008. ATLAS Collaboration, ATLAS-CONF-2012-013.

[7] E. Gabrielli, B. Mele and M. Raidal, arXiv:1202.1796 [hep-ph].

[8] P. P. Giardino, K. Kannike, M. Raidal and A. Strumia, arXiv:1203.4254 [hep-ph].

[9] D. Carmi, A. Falkowski, E. Kuflik and T. Volansky, arXiv:1202.3144. A. Azatov, R. Contino and J. Galloway, arXiv:1202.3415. J. R. Espinosa, C. Grojean, M. Muhlleitner and M. Trott, arXiv:1202.3697.

[10] E. Gabrielli and B. Mele, Phys. Rev. D 82, 113014 (2010) [Erratum-ibid. D 83, 079901 (2011)] [arXiv:1005.2498 [hep-ph]]. E. Gabrielli and B. Mele, Phys. Rev. D 83, 073009 (2011) [arXiv:1102.3361 [hep-ph]]. E. Gabrielli and B. Mele, arXiv:1112.5993 [hep-ph].

[11] M. Kadastik, K. Kannike, A. Racioppi and M. Raidal, arXiv:1112.3647. M. Gonderinger, H. Lim and M. J. Ramsey-Musolf, arXiv:1202.1316 [hep-ph]. J. Elias-Miro, J. R. Espinosa, G. F. Giudice, H. M. Lee and A. Strumia, arXiv:1203.0237 [hep-ph]. O. Lebedev, arXiv:1203.0156 [hep-ph]. C. -S. Chen and Y. Tang, arXiv:1202.5717 [hep-ph]. C. Cheung, M. Papucci and K. M. Zurek, arXiv:1203.5106 [hep-ph].

[12] For a review of the MSSM phenomenology see, A. Djouadi, Phys. Rept. 459 (2008) 1 [hep$\mathrm{ph} / 0503173]$.

[13] For MSSM studies in the context of $125 \mathrm{GeV}$ Higgs boson see references in [2].

[14] For a review of the NMSSM phenomenology see, U. Ellwanger, C. Hugonie and A. M. Teixeira, Phys. Rept. 496 (2010) 1 [arXiv:0910.1785 [hep-ph]]. M. Maniatis, Int. J. Mod. Phys. A 25 (2010) 3505 [arXiv:0906.0777 [hep-ph]].

[15] For NMSSM studies in the context of $125 \mathrm{GeV}$ Higgs boson see references in [2].

[16] R. Barbieri and A. Strumia, [hep-ph/0007265].

[17] M. Kadastik, K. Kannike, A. Racioppi and M. Raidal, Phys. Rev. Lett. 104 (2010) 201301 [arXiv:0912.2729 [hep-ph]]. 
[18] N. Arkani-Hamed and S. Dimopoulos, JHEP 0506 (2005) 073 [hep-th/0405159]. G. F. Giudice and A. Romanino, Nucl. Phys. B 699 (2004) 65 [Erratum-ibid. B 706 (2005) 65] [hep$\mathrm{ph} / 0406088]$.

[19] R. Aaij et al. [LHCb Collaboration], arXiv:1203.4493 [hep-ex]. CMS Collaboration, Summary plot at CMS Wiki.

[20] J. Hisano, K. Ishiwata and N. Nagata, Phys. Lett. B 690 (2010) 311 [arXiv:1004.4090 [hep-ph]].

[21] G. F. Giudice, C. Grojean, A. Pomarol and R. Rattazzi, JHEP 0706 (2007) 045 [arXiv:hepph/0703164]. R. Contino, C. Grojean, M. Moretti, F. Piccinini and R. Rattazzi, JHEP 1005, 089 (2010) [arXiv:1002.1011]. R. Grober and M. Muhlleitner, JHEP 1106, 020 (2011) [arXiv:1012.1562]. S. De Curtis, M. Redi and A. Tesi, arXiv:1110.1613.

[22] M. S. Carena, J. R. Espinosa, M. Quiros and C. E. M. Wagner, Phys. Lett. B 355 (1995) 209 [hep-ph/9504316].

[23] E. Gabrielli, K. Kannike, B. Mele, A. Racioppi and M. Raidal, arXiv:1204.0080 [hep-ph].

[24] M. Raidal and A. Strumia, Phys. Rev. D 84 (2011) 077701 [arXiv:1108.4903]. Y. Mambrini, Phys. Rev. D 84 (2011) 115017 [arXiv:1108.0671]. J. F. Kamenik and C. Smith, arXiv:1201.4814 [hep-ph]. A. Arhrib, R. Benbrik and N. Gaur, arXiv:1201.2644 [hep-ph]. X. -G. He, B. Ren and J. Tandean, arXiv:1112.6364 [hep-ph]. C. Cheung and Y. Nomura, arXiv:1112.3043 [hep-ph]. X. Chu, T. Hambye and M. H. G. Tytgat, 1112.0493 [hep-ph]. O. Lebedev, H. M. Lee and Y. Mambrini, Phys. Lett. B 707 (2012) 570 [arXiv:1111.4482 [hep-ph]]. C. Englert, J. Jaeckel, E. Re and M. Spannowsky, Phys. Rev. D 85 (2012) 035008 [arXiv:1111.1719 [hep-ph]]. I. Low, P. Schwaller, G. Shaughnessy and C. E. M. Wagner, Phys. Rev. D 85 (2012) 015009 [arXiv:1110.4405 [hep-ph]]. M. Pospelov and A. Ritz, Phys. Rev. D 84 (2011) 113001 [arXiv:1109.4872 [hep-ph]]. T. Cohen, J. Kearney, A. Pierce and D. TuckerSmith, arXiv:1109.2604 [hep-ph]. X. -G. He and J. Tandean, Phys. Rev. D 84 (2011) 075018 [arXiv:1109.1277 [hep-ph]]. Y. Mambrini, Phys. Rev. D 84 (2011) 115017 [arXiv:1108.0671 [hep-ph]]. C. -S. Chen and Y. Tang, arXiv:1202.5717 [hep-ph]. V. Barger, M. Ishida and W. Y. Keung, arXiv:1203.3456. J. Cao, Z. Heng, J. M. Yang and J. Zhu, arXiv:1203.0694 [hepph]. A. Djouadi, O. Lebedev, Y. Mambrini and J. Quevillon, Phys. Lett. B 709 (2012) 65 [arXiv:1112.3299]. V. Barger, M. Ishida and W. -Y. Keung, arXiv:1203.3456 [hep-ph]. 